\title{
Factors Controlling Reactivity in the Hydrogen Atom Transfer and Radical Addition Steps of a Radical Relay Cascade
}

\author{
Yike Zou ${ }^{\dagger}$, Xiao-Song Xue $^{\dagger}$, Yifan Deng ${ }^{\ddagger}$, Amos B. Smith III ${ }^{\ddagger}$, K. N. Houk ${ }^{*} \dagger$ \\ tDepartment of Chemistry and Biochemistry, University of California, Los Angeles, California \\ 90095-1569, United States \\ ‡Department of Chemistry, University of Pennsylvania, 231 South 34th Street, Philadelphia, \\ Pennsylvania 19104, United States
}

\begin{abstract}
DFT exploration of 1,5- and 1,6-hydrogen atom transfers (HAT), radical addition, and relay to alkene is reported. The reactivity of 1,5- and 1,6-HAT are similar. We also explored reactions involving a multiple radical transferring process. Initially generated radicals undergo a multiple HAT process to form multiple bonds in a "relay" fashion, provided by exquisitely balanced rates.
\end{abstract}

\section{Graphical Abstract}

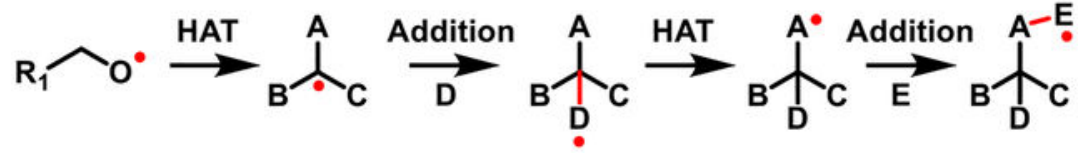

The hydrogen atom transfer (HAT) reaction transfers a radical center from the original site to a distal position. ${ }^{1}$ Although the newly generated radical specie is less reactive than the original one, a new bond can now be formed at the distal position, if the lifetime is sufficient for a subsequent addition reaction. One of the earliest examples of a HAT reaction is the Hofmann-Löffler-Freytag reaction, ${ }^{2}$ in which the initial nitrogen based radical, generated via photolytic homolysis of the $\mathrm{N}-\mathrm{Cl}$ bond such as in (i), undergoes an intramolecular HAT process to relay the radical to a carbon center (Scheme 1) that subsequently reacts with the amine generated to give (ii). In the Norrish Type II reaction, ${ }^{3}$ a carbonyl such as in (iii) excited to an $n-\pi^{*}$ state undergoes rapid intramolecular HAT to abstract a $\gamma$-hydrogen to produce a 1,4-biradical as the primary photoproduct. The resulting 1,4-biradical then undergoes subsequent reactions of intra-molecular radical recombination [Yang cyclization to generate (iv)] and/or $\beta$-cleavage (Norrish Type II).

\footnotetext{
*Corresponding Author: houk@chem.ucla.edu.

Supporting Information

The Supporting Information is available free of charge on the ACS Publications website at DOI: 10.1021/acs.orglett.9b02023. General computational methods; energetics of all computed species; Cartesian coordinates of all computed species; references to computational program (PDF)

The authors declare no competing financial interest.
} 
The most common synthetic applications of HAT involve 1,5- and 1,6-HAT reactions, wherein the newly generated radical-bearing atoms are in a 1,5- and 1,6-relationship respectively with the original radical centers. ${ }^{4}$ Smith et al. recently reported a new synthetic application of the 1,5- and 1,6-HAT process in which an initial alkoxy radical (vi) was generated from a $N$-alkoxyphthalimide (v) via photoredox catalysis (Scheme 1). Upon HAT, the resulting carbon radical (vii), stabilized by either a dioxanyl or a dithianyl group, further engages in radical additions to electrophiles. ${ }^{5}$ This radical "relay" reaction permits remote $\mathrm{C}-\mathrm{H}$ functionalization and was employed in the total synthesis of $( \pm)-$ danshenspiroketallactone (x). The originally generated oxy radical reacts in an energetic cascade with a series of electrophiles to generate multiple bonds in a single-flask operation. This work demonstrated the synthetic potential of a radical cascade reaction. ${ }^{6}$

Previously we reported computational studies of intramolecular 1,5- and 1,6-HAT reactions that demonstrate that the 1,5-HAT process is in general more feasible than the 1,6-HAT process. ${ }^{7}$ That is, a six-membered cyclic transition state is both thermodynamically more stable than a seven-membered cyclic transition state and entropically more favored. $A b$ initio calculations of the intramolecular HAT reactions of butan-1-oxyl and pentan-1-oxyl radicals at the MP2/6-31G(d)//UHF/3-21G level demonstrated that the 1,5-HAT possessed a 19.9 $\mathrm{kcal} / \mathrm{mol}$ energy barrier, and the barrier of the $1,6-\mathrm{HAT}$ was $21.7 \mathrm{kcal} / \mathrm{mol}{ }^{4}{ }^{4}$ This 1.8 $\mathrm{kcal} / \mathrm{mol}$ free energy difference was introduced not from enthalpy $\left(\Delta \Delta H^{+}=-0.7 \mathrm{kcal} / \mathrm{mol}\right)$ but from entropy $\left(\Delta \Delta \mathrm{S}^{\ddagger}=8.3 \mathrm{eu}\right.$, corresponding to $2.5 \mathrm{kcal} / \mathrm{mol}$ at $\left.\mathrm{rt}\right) .{ }^{4}$ Development of DFT methods have now provided an opportunity to reinvestigate the 1,5 - and 1,6-HAT process at a more superior computational level and to provide new mechanistic insights.

The structures of the buta-1-oxyl radical (1) and pentan-1-oxyl radical (2) were optimized using the DFT method at the uM062X(D3)/6-311++G(2d,p)//uM062X(D3)/6-31G(d) level (Scheme 2). ${ }^{8}$ Each corresponding reactive conformation (2 and 6) led to an intramolecular HAT cyclic transition state (TS1 and TS2) to generate a primary carbon radical (4 and $\mathbf{8}$ ). The energy barriers $(11.4$ and $11.7 \mathrm{kcal} / \mathrm{mol})$ for the HAT processes are significantly lower compared to our previous calculations.

We found that activation enthalpies and entropies of 1,5-and 1,6-HAT transition states are similar to each other. Seven-membered transition states generally possess more low energy conformations than six-membered transition states, but the seven-membered cyclic transition state of the HAT process possesses almost the same free energy as the six-membered cyclic transition state.

With this principle in mind, we continued our investigations by introducing either a dioxolanyl (9a and $\mathbf{9 b}$ ) or a dithiolanyl (9c and $9 \mathbf{d}$ ) as the radical stabilizing group at the HAT destination (Scheme 3). Not surprisingly, the resulting dioxolanyl (11a and 11b) and dithiolanyl (11c and 11d) radicals in both 1,5- and 1,6-HAT processes have significant thermodynamic preferences. Interestingly, both the 1,5- and 1,6-HAT are also kinetically more facile than those without the radical stabilizing groups (see Scheme 2), as can be demonstrated by their low free energy barriers [see TS3 (a-d), Scheme 3]. This result is due to the radical stabilization effect of the dioxolanyl and dithiolanyl groups, and also to the 
Thorpe-Ingold effect that stabilizes the cyclic conformations, which together resulted in a lower barrier compared with the previous examples (see Scheme 2).

The second relay step (a.k.a. radical addition onto the electrophile) was also investigated. Upon introduction of an allyl sulfone electrophile 12 (Scheme 3), the dioxolanyl (11a and 11b) and dithiolanyl (11c and 11d) radicals underwent conjugate addition followed by an $a$ fragmentation/elimination of $\mathbf{1 3}$ to generate the corresponding allylic substituted products $\mathbf{1 4}$ and a sulfonyl radical 15. The radical addition step for dioxolanyl radicals proceeded with a free energy barrier of only 9.6 (TS4a, 1,5-HAT) or 8.5 (TS4b, 1,6-HAT) kcal/mol, which is comparable to the barrier of the HAT process $(7.0 \mathrm{kcal} / \mathrm{mol}$ for $1,5-\mathrm{HAT}$ and $7.8 \mathrm{kcal} / \mathrm{mol}$ for 1,6-HAT). However, the free energy barrier for the radical addition steps of the dithiolanyl radicals were significantly higher $[12.9 \mathrm{kcal} / \mathrm{mol}$ for the $1,5-\mathrm{HAT}(\mathbf{T S 4 c})$ and $13.6 \mathrm{kcal} / \mathrm{mol}$ for the 1,6-HAT (TS4d)], with the conjugate addition step now determining the rate of the overall reaction. This higher energy barrier explains in part why a dithiolane substrate resulted in a lower yield in the experiments. The increased reaction barrier of the second step is likely due to the stability of a dithiolanyl radical. The final $a$-fragmentation/ elimination is in equilibrium, and depending on reaction conditions this might permit a further cascade reaction to occur. This proved to be the case as it was possible to introduce multiple relay steps after the initial HAT, as demonstrated in the original report. ${ }^{5}$

In the following example (Scheme 4) upon the first radical addition that generates tertiary radical 18a, the tertiary radical undergoes a 1,6-HAT to transport the single electron to the benzylic position of $\mathbf{1 9}$, followed by adding to the second equivalent of $\mathbf{1 2}$. An elimination then occurs that leads to a double addition adduct 20 in $56 \%$ overall yield after oxidation. ${ }^{5}$ We have now investigated this relay process computationally. Upon generation of benzylalkoxy radical 16, a facile 1,5-HAT (TS5, $5.9 \mathrm{kcal} / \mathrm{mol}$, Scheme 5) generates benzylic radical 17. Addition to the radical acceptor 12 leads to $18 \mathbf{a}$ that is in equilibrium with $\mathbf{1 8 b}$. 18a undergoes a seven-membered 1,6-HAT transition state (TS7) and then generates a benzylic radical (19). Final addition of another equivalent of $\mathbf{1 2}$ followed by $a$ fragmentation furnishes the final product $\mathbf{2 0 b}$. The 1,6-HAT process is now the rate-limiting step $(16.8 \mathrm{kcal} / \mathrm{mol}$ free energy barrier) in this "energetic cascade". The multiple migration of the original radical from oxy- to dithiolanyl-, $\beta$-sulfonyl-, benzyl-, $\beta$-sulfonyl-, and finally sulfonyl- are accompanied by bond relocation.

In summary, 1,5- and 1,6-HAT reactions have been further investigated using DFT methods and as shown have similar reactivity. Importantly, installation of radical stabilizing groups leads to the generation of a multiple-stage process involving multiple chemical bond formations. In order to achieve this energetic cascade, electrophiles must be selected in a manner that a radical can be generated at a new position upon each transfer without being quenched. Via this concept, multiple bond construction can be achieved in a single-flask in a "relay" manner, to facilitate the construction of complex molecular structures.

\section{Supplementary Material}

Refer to Web version on PubMed Central for supplementary material. 


\section{ACKNOWLEDGMENTS}

We acknowledge the National Science Foundation (CHE-1764328), the National Institutes of Health, National Institute of General Medical Sciences (R01 GM109078), for financial support of this research to K.N.H. Computation time was provided by the UCLA Institute for Digital Research and Education (IDRE).

\section{REFERENCES}

(1). Huynh MHV; Meyer TJ Proton-Coupled Electron Transfer. Chem. Rev 2007, 107, 5004-5064. [PubMed: 17999556]

(2). (a)Hofmann AW Ueber die Einwirkung des Broms in alkalischer Lösung auf die Amine. Ber. Dtsch. Chem. Ges 1883, 16, 558-560.(b)Löffler K; Freytag C Über eine neue Bildungsweise von N-alkylierten Pyrrolidinen. Ber. Dtsch. Chem. Ges 1909, 42, 3427-3431.(c)Stella L Homolytic Cyclizations of N-Chloroalkenylamines. Angew. Chem., Int. Ed. Engl 1983, 22, 337-350. (d)Majetich G; Wheless K Remote intramolecular free radical functionalizations: An update. Tetrahedron 1995, 51, 7095-7129.

(3). (a)Norrish RGW; Bamford CH Photo-decomposition of Aldehydes and Ketones. Nature 1937, 140, 195-196.(b)Pitts JN; Blacet FE METHYL ETHYL KETONE PHOTOCHEMICAL PROCESSES. J. Am. Chem. Soc 1950, 72, 2810-2811.(c)Paquette LA; Ternansky RJ; Balogh DW A strategy for the synthesis of monosubstituted dodecahedrane and the isolation of an isododecahedrane. J. Am. Chem. Soc 1982, 104, 4502-4503.(d)Ternansky RJ; Balogh DW; Paquette LA Dodecahedrane. J. Am. Chem. Soc 1982, 104, 4503-4504.(e)Paulson SE; Liu D-L; Orzechowska GE; Campos LM; Houk KN Photolysis of Heptanal. J. Org. Chem 2006, 71, 64036408. [PubMed: 16901122] (f)Schwinden MD The Norrish Type II reaction in organic synthesis Ph.D. Thesis, Iowa State University, 1990.

(4). (a)Dorigo AE; McCarrick MA; Loncharich RJ; Houk KN Transition structures for hydrogen atom transfers to oxygen. Comparisons of intermolecular and intramolecular processes, and open- and closed-shell systems. J. Am. Chem. Soc 1990, 112, 7508-7514.(b)Shtarev AB; Dolbier WR; Smart BE Rates of 1,5-Hydrogen Abstraction in 1,1,2,2-Tetrafluoro-n-pentyl and 1,1,2,2,3,3Hexafluoro-n-pentyl Radicals. J. Am. Chem. Soc 1999, 121, 2110-2114.(c)Wagner PJ 1,5Biradicals and five-membered rings generated by.delta.-hydrogen abstraction in photoexcited ketones. Acc. Chem. Res 1989, 22, 83-91.

(5). Deng Y; Nguyen MD; Zou Y; Houk KN; Smith AB Generation of Dithianyl and Dioxolanyl Radicals Using Photoredox Catalysis: Application in the Total Synthesis of the Danshenspiroketallactones via Radical Relay Chemistry. Org. Lett 2019, 21, 1708-1712. [PubMed: 30807194]

(6). Plesniak MP; Huang H-M; Procter DJ Radical cascade reactions triggered by single electron transfer. Nature Reviews Chemistry 2017, 1, 0077.

(7). (a)Dorigo AE; Houk KN Transition structures for intramolecular hydrogen-atom transfers: the energetic advantage of seven-membered over six-membered transition structures. J. Am. Chem. Soc 1987, 109, 2195-2197.(b)Dorigo AE; Houk KN The relationship between proximity and reactivity. An ab initio study of the flexibility of the $\mathrm{OH}$.bul. $+\mathrm{CH} 4$ hydrogen abstraction transition state and a force-field model for the transition states of intramolecular hydrogen abstractions. J. Org. Chem 1988, 53, 1650-1664.

(8). All calculations were performed using Gaussian 09, Revision A.02 (for full citation, please see Supporting Information). 
Hofmann-Löffler-Freytag reaction example<smiles>CCNCCCN1CCCCC1CCN1CCCCC1C</smiles>

(i)

(ii)

Norrish reaction - type II example<smiles>C=C(C)[C@]1(C)CC[C@@H](C)CC1=O</smiles>

(iii)<smiles>C=C1CC2(O)C[C@H](C)CC[C@]12C</smiles>

(iv)

Radical Relay (Smith et al., 2019)

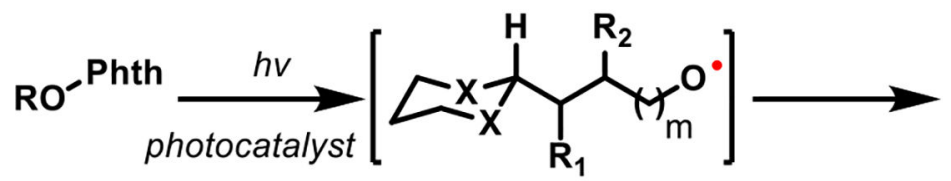

(v) $\mathrm{m}=1$ or $2 \mathrm{X}=\mathrm{O}$ or $\mathrm{S}$

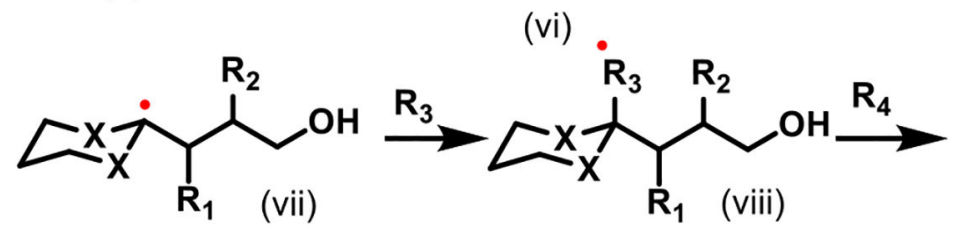

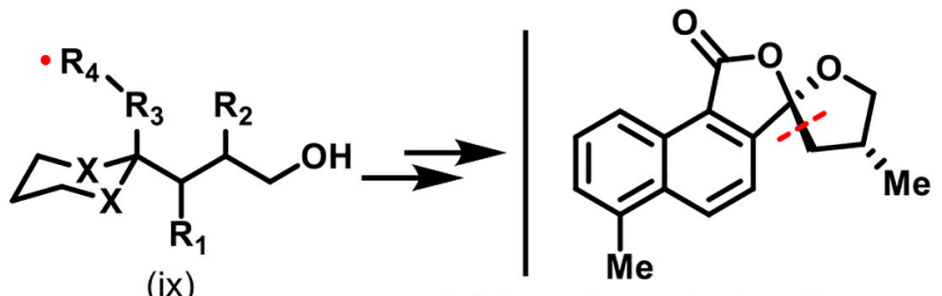

(士)-Danshenspiroketallactone $(x)$

Scheme 1.

Demonstration of Intramolecular HAT Reactions 


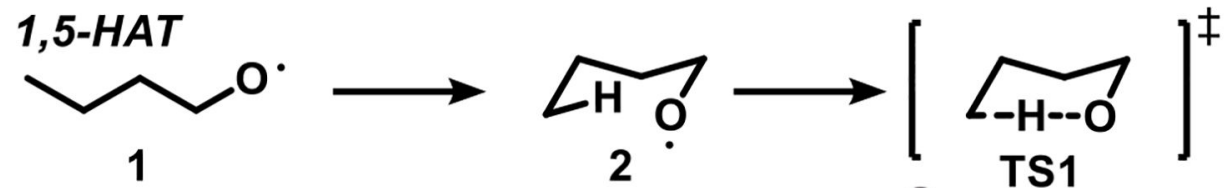

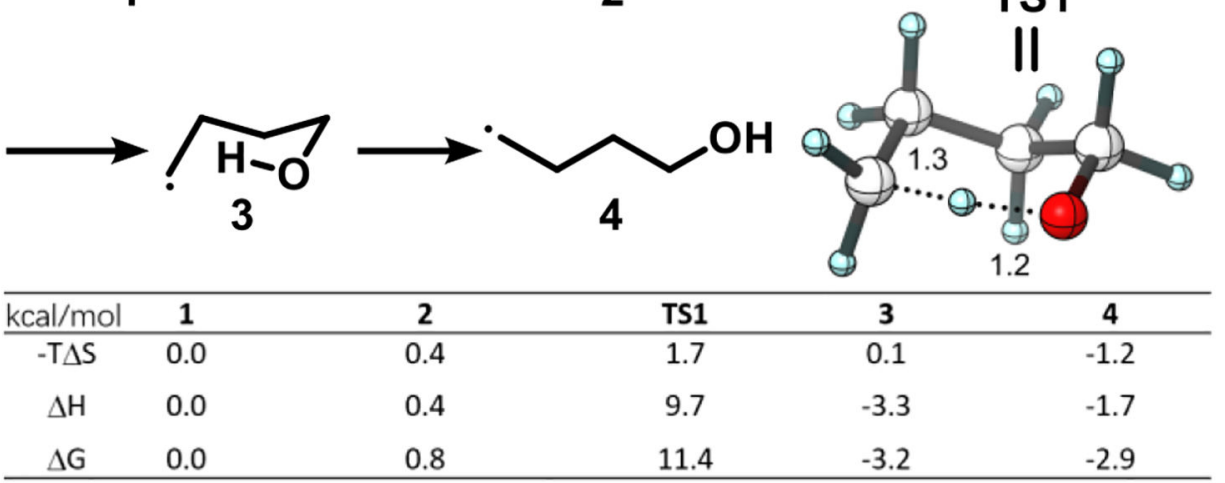

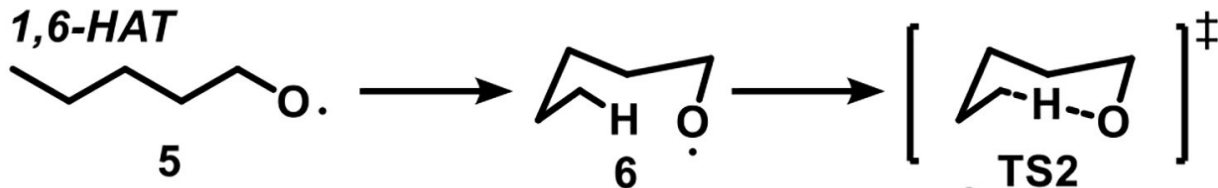

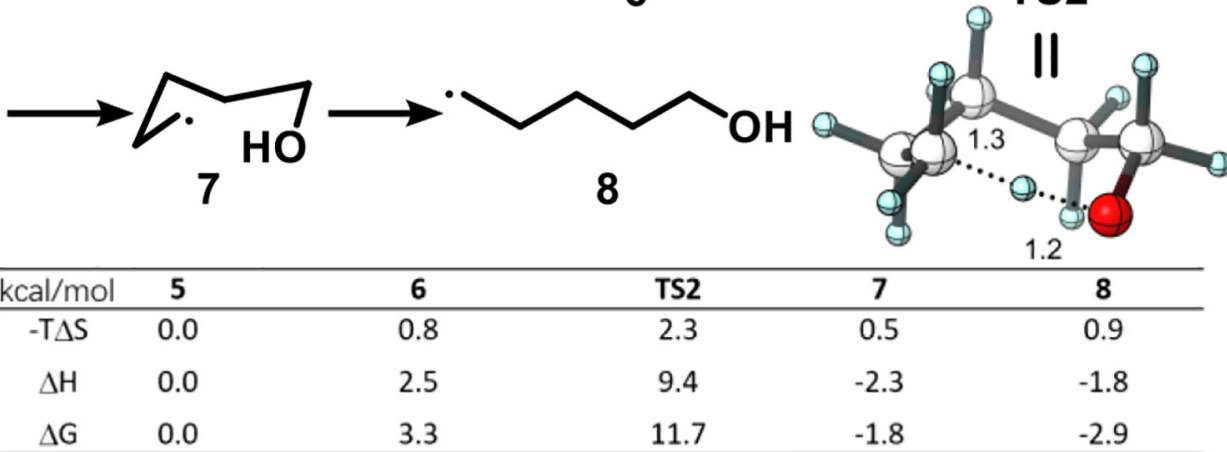

Scheme 2.

Energetics of HAT Reactions Using DFT Methods 


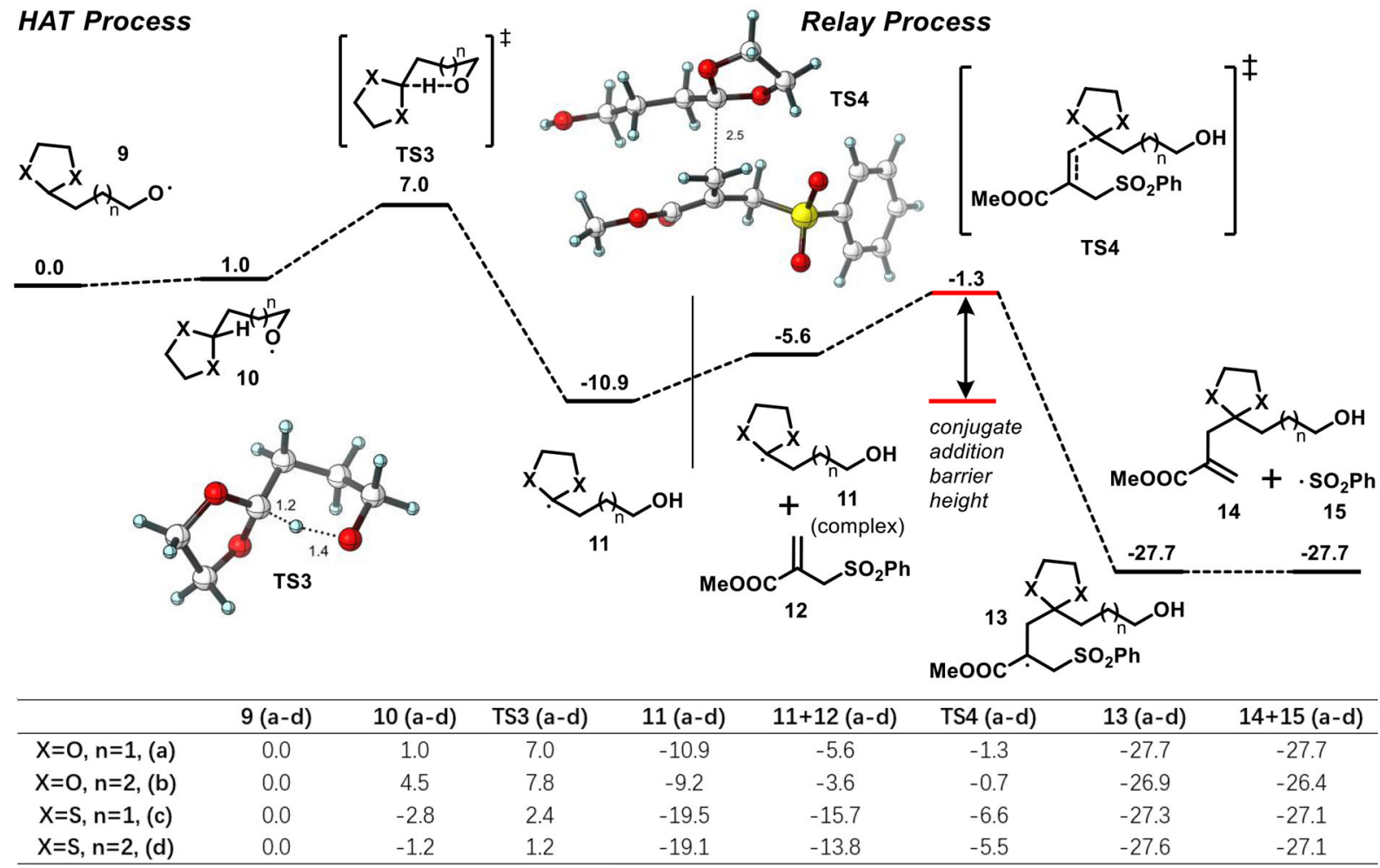

Scheme 3.

Free Energy Profile (298 K, 1 atm) of the Radical Relay Reactions 

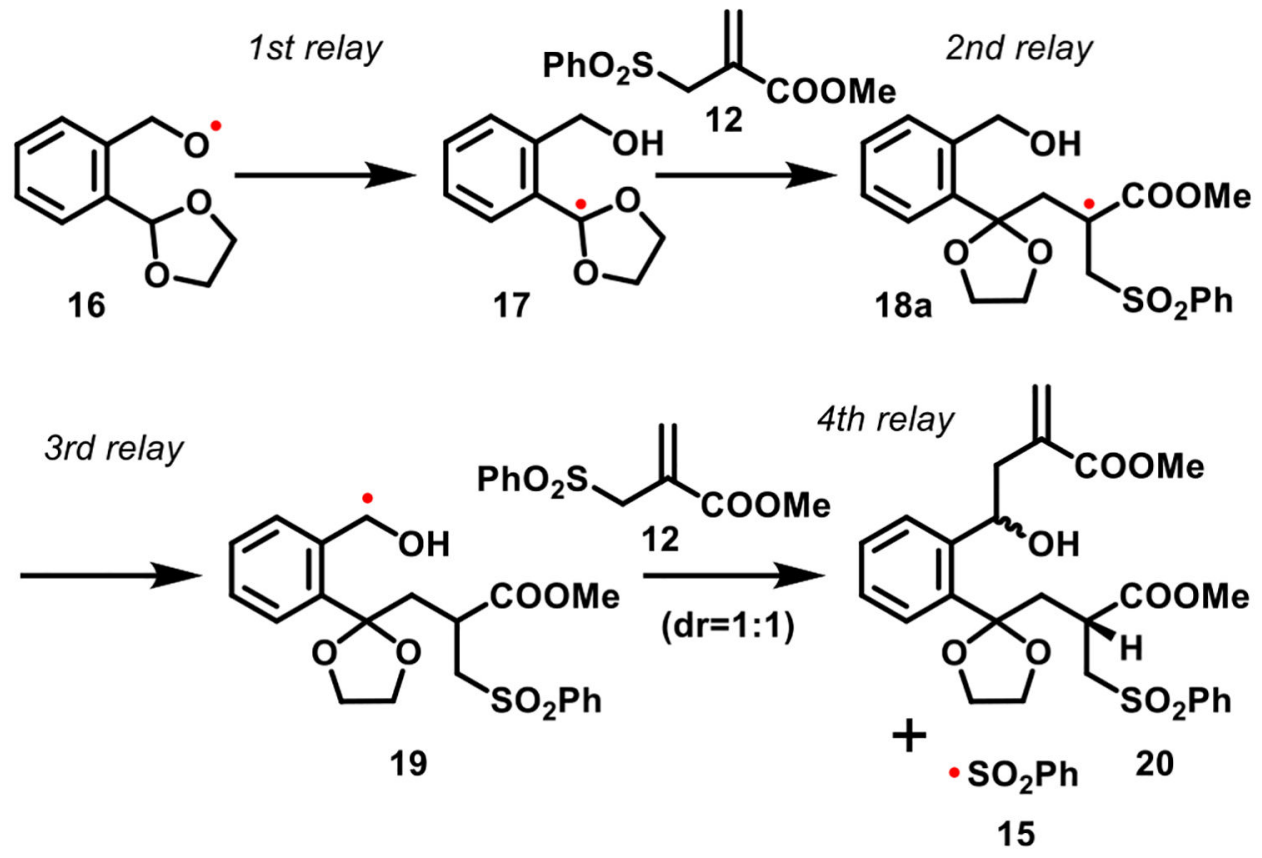

Scheme 4.

Multiple Relay Reaction 


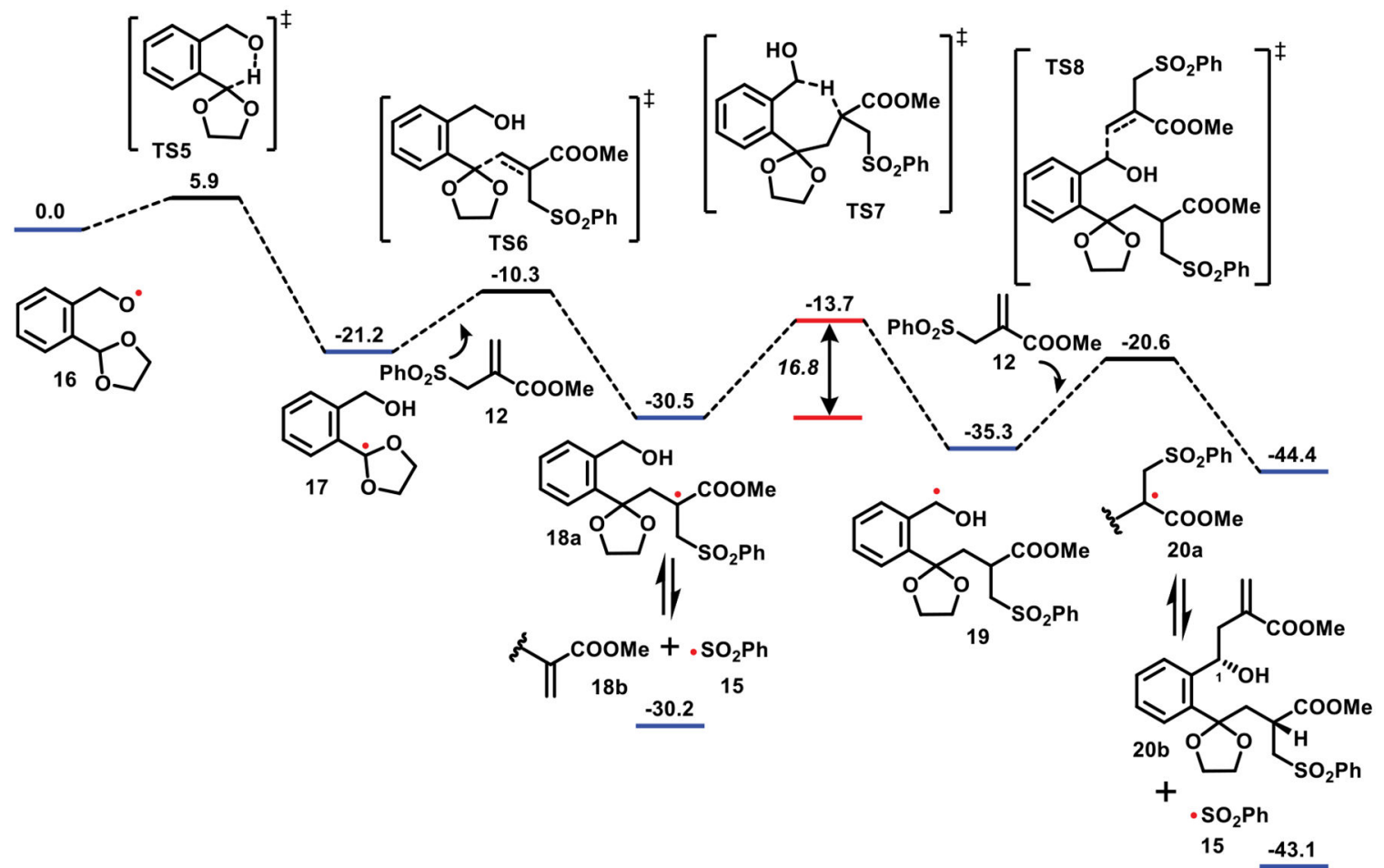

Scheme 5.

Free Energy Profile for the Quadruple-Relay Process (298 K, 1 atm) 